Received: $\quad 2019.09 .29$

Accepted: 2019.10 .22 Available online: 2020.01 .21 Published: 2020.02.11

\title{
Pleuromutilin Inhibits Proliferation and Migration of A2780 and Caov-3 Ovarian Carcinoma Cells and Growth of Mouse A2780 Tumor Xenografts by Down-Regulation of pFAK2
}

Authors' Contribution: Study Design A Data Collection B Statistical Analysis C Data Interpretation D Manuscript Preparation E Literature Search F Funds Collection G
BCDE 1 Bo Zhang

CEF 1 Xiaoli Ma

BCD 2 Yuan Li

CDE 3 Sijing Li

ADEF 1 Jiumei Cheng
1 Department of Minimally Invasive Gynecological Centre, Beijing Obstetrics and Gynecology Hospital, Capital Medical University, Beijing, P.R. China 2 Department of Perinatal Medicine, Beijing Obstetrics and Gynecology Hospital, Capital Medical University, Beijing, P.R. China

3 Department of Ultrasound, Beijing Obstetrics and Gynecology Hospital, Capital Medical University, Beijing, P.R. China
Corresponding Author: Source of support:

Background: Pleuromutilin is a natural tricyclic, derived from the fungus, Pleurotus mutilus. This study aimed to investigate the effects of pleuromutilin on migration and proliferation of A2780 and Caov-3 human ovarian carcinoma cells and the growth of A2780 tumor xenografts in mice and the molecular mechanisms involved.

Material/Methods: A2780 and Caov-3 human ovarian carcinoma cells were cultured with and without 40, 160, and $200 \mu \mathrm{M}$ of pleuromutilin. The Edu fluorescence assay, the wound-healing assay, and Matrigel were used to measure A2780 and Caov-3 cell proliferation, migration, invasion, and adhesion in vitro, respectively. Western blot measured protein levels of FAK, p-FAK, MMP-2, and MMP-9. A2780 cells were injected subcutaneously into mice to determine the effects of pleuromutilin on the growth of tumor xenografts.

Results: Pleuromutilin significantly reduced A2780 and Caov-3 cell proliferation at $48 \mathrm{~h}$ in a dose-dependent manner $(\mathrm{P}<0.05)$, and at $200 \mu \mathrm{M}$, pleuromutilin reduced cell proliferation by $21.43 \%$ and $23.65 \%$, respectively. Treatment of A2780 cells with pleuromutilin significantly reduced cell migration, invasion, and adhesion and the expression of p-FAK, MMP-2, and MMP-9 compared with untreated controls. In the mouse tumor xenograft model, treatment with pleuromutilin significantly reduced tumor size compared with the untreated group and inhibited tumor metastasis to the intestine, spleen, and peritoneal cavity.

Conclusions: In A2780 and Caov-3 human ovarian carcinoma cells, pleuromutilin inhibited cell proliferation, migration, invasion, and adhesion in a dose-dependent manner, and reduced tumor growth and metastases in a mouse A2780 cell tumor xenograft model.

MeSH Keywords: $\quad$ Adhesins, Bacterial • Lymphatic Metastasis • Matrix Metalloproteinase 1

Full-text PDF: https://www.medscimonit.com/abstract/index/idArt/920407

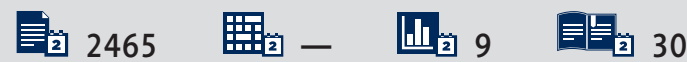




\section{Background}

Worldwide, ovarian carcinoma has a high mortality rate and is the seventh most commonly diagnosed malignancy in women [1]. The incidence of ovarian cancer has increased during the past few decades [1]. The mortality rate from ovarian cancer remains high due to the lack of early diagnosis and the lack of effective treatments for late-stage malignancy [2]. Women who present with late-stage ovarian carcinoma continue to have a poor prognosis [1]. Clinically, ovarian carcinoma is divided into several subtypes and high-grade and lowgrade carcinoma based on tumor histopathology [3].

Ovarian carcinoma commonly metastasizes to the peritoneal cavity and results in 5-year survival of less than 30\% [4]. Worldwide, ovarian carcinoma has the highest patient mortality rate of all gynecological malignancy [3]. Advances in treatment, including chemotherapy, has resulted in the development of effective treatment approaches based on targeted molecular therapy [5-7]. There have been recent developments in targeted therapies and immunotherapy in ovarian carcinoma [8]. Recent studies have investigated the role of bevacizumab and nivolumab in ovarian carcinoma, with the aim of developing effective treatment $[9,10]$.

Pleuromutilin is a natural tricyclic, derived from the fungus, Pleurotus mutilus. Pleuromutilin was first isolated in 1951 and consisted of a tricyclic 5-6-8 member diterpenoid structure [11]. Pleuromutilin has shown significant activity against Gram-positive bacteria in vitro, but these findings have not been supported clinically [12]. Mechanistic studies have shown that pleuromutilin interacts with the ribosomes of prokaryotic organisms and inhibit the synthesis of proteins $[13,14]$. Changes in the structure of pleuromutilin by chemical modification has been undertaken to develop C-14 acyloxy-derived compounds (Figure 1) $[15,16]$. The modification at C-14 of pleuromutilin has resulted in the development of an oral antibiotic, tiamulin, which is currently used in veterinary practice $[17,18]$. However, there have been no previous studies on the effects of pleuromutilin on human malignant cells in vitro.

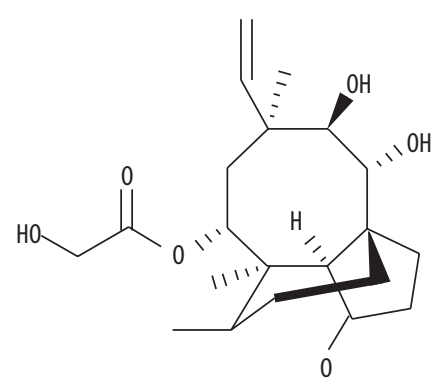

Figure 1. The chemical structure of pleuromutilin.
Therefore, this study aimed to investigate the effects of pleuromutilin on migration and proliferation of A2780 and Caov-3 human ovarian carcinoma cells and the growth of A2780 tumor xenografts in mice and the molecular mechanisms involved.

\section{Material and Methods}

\section{Cell culture}

A2780 and Caov-3 cells were obtained from the Cell Bank, Chinese Academy of Sciences, Shanghai, China. The cells were cultured in RPMI-1640 (Gibco, Thermofisher Scientific, Waltham, MA, USA) medium containing 10\% fetal bovine serum (Gibco, Thermofisher Scientific, Waltham, MA, USA) at a temperature of $37^{\circ} \mathrm{C}$ under an atmosphere of $5 \% \mathrm{CO}_{2}$.

\section{The Edu fluorescence cell proliferation assay}

A2780 and Caov-3 cell proliferation after 48 of treatment with pleuromutilin was determined using Edu proliferation assay in accordance with the manufacturer's instructions. The cells were distributed at $3 \times 10^{4}$ cells/well density in 96-well plates and cultured for $24 \mathrm{~h}$. The medium was changed by fresh medium mixed with $10,20,40,80,160$, and $200 \mu \mathrm{M}$ of pleuromutilin, and incubation was performed for $48 \mathrm{~h}$. Cell proliferation was determined using an Edu proliferation assay kit (Guangzhou RiboBio Co., Ltd., Guangzhou, China). An Olympus IX51 fluorescence microscope (Olympus Corporation, Tokyo, Japan) was used to observe the stained cells.

\section{Cell cycle and apoptosis analysis}

Flow cytometry was used for the analysis of apoptosis in A2780 and Caov-3 cells. Briefly, the cells were put into 6-well plates at $2 \times 10^{5}$ cells/well density and exposed for $48 \mathrm{~h}$ to 40,160 , and $200 \mu \mathrm{M}$ pleuromutilin. The cells in log phase were harvested by centrifugation and subsequently washed two times with ice-cold PBS. Then the cells were resuspended in PBS followed by fixing with cold $75 \%$ ethyl alcohol at $4^{\circ} \mathrm{C}$ overnight. The cells were stained with Annexin-V/propidium iodide (PI) and examined using a BD FACSVantage SE flow cytometer (Becton-Dickinson, San Jose, CA, USA) was used to identify cell apoptosis.

\section{Cell adhesion assay}

Matrigel (BD Biosciences, Shanghai, China) coated 96-well culture plates were treated for $1.5 \mathrm{~h}$ with $1 \%$ bovine serum albumin (BSA) at room temperature to block the non-specific binding sites. A2780 and Caov-3 cells were incubated for $48 \mathrm{~h}$ with 40,160 , and $200 \mu \mathrm{M}$ of pleuromutilin. The cells at $3 \times 10^{4}$ density were cultured in the plates at $37^{\circ} \mathrm{C}$ and allowed to adhere 
for $2 \mathrm{~h}$ to the Matrigel-coated wells. The cells which did not adhere to the wells were removed by washing the plates with cold PBS three times. The cell adhesion was determined by comparing the adherent cell population in pleuromutilin treated plates with the control.

\section{Analysis of cell invasion}

The invasion of A2780 cells was determined using a Boyden chamber. The cells at a density of $3 \times 10^{4}$ cells $/ \mathrm{ml}$ were collected by trypsinization and then resuspended in culture medium without serum. The cells at $2 \times 10^{5}$ cells $/ \mathrm{ml}$ concentration were then put into the upper compartment of the Boyden chamber. Pleuromutilin at increasing concentrations was added to the inner well of the Boyden chamber pre-coated with $45 \mu \mathrm{l}$ of Matrigel (at dilution of 1: 6 in serum-free medium). The lower chamber contained RPMI-1640 medium mixed with $20 \%$ fetal bovine serum (FBC). The invasion potential of the cells into the Matrigel was assessed following $48 \mathrm{~h}$ of incubation at $37^{\circ} \mathrm{C}$. The invaded cells were fixed with methyl alcohol and stained using hematoxylin and eosin (H\&E). Cotton swabs removed the non-invaded cells in the upper chamber. Cell invasion was determined using light microscopy.

\section{Cell migration assay}

The migration potential of A2780 cells after treatment with pleuromutilin was assessed using the wound-healing assay. The cells were cultured in the six-well plate at a density of $2 \times 10^{5}$ cells/well, and incubated with 40,160 , and $200 \mu \mathrm{M}$ of pleuromutilin for $48 \mathrm{~h}$. The monolayer of cells was scratched using a plastic cell scraper, followed washing three times with PBS to remove the non-adherent cells. The adhered cells were incubated for $48 \mathrm{~h}$ in a humid atmosphere of $5 \% \mathrm{CO}_{2}$ at $37^{\circ} \mathrm{C}$. Cell migration was determined by assessing the cell counts in the scratched zone.

\section{Western blot}

A2780 cells were treated with 40,160 , and $200 \mu \mathrm{M}$ of pleuromutilin for $48 \mathrm{~h}$, and then harvested after washing in cold PBS. The cells were treated with RIPA lysis buffer (Roche, Shanghai, China) for $45 \mathrm{~min}$ on ice. The lysate was centrifuged at $4^{\circ} \mathrm{C}$ at $12,000 \mathrm{rpm}$ for $15 \mathrm{~min}$ to isolate the supernatant. The concentration of proteins in the supernatant was determined by the Bradford method. The proteins were transferred onto the membrane (Merck Millipore, Burlington, MA, USA) and separated on an $8-12 \%$ sodium dodecyl sulfate-polyacrylamide gel electrophoresis (SDS-PAGE) gel. Non-specific binding sites were blocked by a $2 \mathrm{~h}$ incubation with $10 \%$ dried skimmed milk powder in TBST at room temperature. Incubation of the membranes was performed overnight at $4^{\circ} \mathrm{C}$ with primary antibodies to p-FAK, MMP-2, FAK, MMP-9, and $\beta$-actin (Cell Signaling
Technology, Shanghai, China). After washing the membranes three times with TBST, the secondary antibodies were applied, and incubation was performed at room temperature for $1 \mathrm{~h}$. The protein expression levels were detected using an electrochemiluminescence (ECL) kit (Merck Millipore, Burlington, MA, USA) and the bands were visualized using autoradiography.

\section{The mouse A2780 cell tumor xenograft model}

Sixty 6-week-old female nude mice were supplied by the Centre for Experimental Animals, Wuhan University, Wuhan, China. The mice were housed in sterilized, air-filtered, pathogenfree cages in the animal center. The temperature was maintained at $23 \pm 2^{\circ} \mathrm{C}$ with a humidity of $65 \%$. The mice had access to water and food ad libitum and were maintained in a 12-hour light and dark cycles. The mice were divided randomly into the untreated control group, and groups treated with 50 , 100,150 , and $200 \mathrm{mg} / \mathrm{kg}$ of pleuromutilin. The mice were inoculated with A2780 human ovarian carcinoma cells at a cell density of $2 \times 10^{6}$ cells in $100 \mu \mathrm{l}$ of PBS on the dorsal side of the body under anesthesia. The solid tumors that formed in the mice were sliced into $1 \mathrm{~mm}^{3}$ thin sections, which were implanted in the ovarian capsule of the mice using laparotomy under anesthesia. The incision in the abdomen was sutured with 3-0 silk after returning the ovary to its original position. On the second day of tumor implantation, the mice were treated with $50,100,150$, and $200 \mathrm{mg} / \mathrm{kg}$ doses of pleuromutilin through the intraperitoneal route. The mice were euthanized on day 30 after tumor implantation to excise the ovarian tumors, and the volume was measured using calipers.

\section{Statistical analysis}

Data were presented as the mean \pm standard deviation (SD). Student's t-test and two-way analysis of variance (ANOVA) were used. Data were analyzed using GraphPad Prism version 4.0 (GraphPad Software, San Diego, CA, USA). A P-value $<0.05$ was considered to be statistically significant.

\section{Results}

\section{Pleuromutilin inhibited A2780 and Caov-3 cell growth}

Pleuromutilin treatment significantly $(P<0.05)$ suppressed the proliferation of A2780 and Caov-3 cells in a dose-dependent manner (Figure 2). The different concentrations of pleuromutilin tested against $\mathrm{A} 2780$ and Caov-3 cells ranged from 0-200 $\mu \mathrm{M}(10,20,40,80,160$, and $200 \mu \mathrm{M})$. Pleuromutilin showed an $\mathrm{IC}_{50}$ of $40 \mu \mathrm{M}$ against $\mathrm{A} 2780$ and Caov-3 human ovarian carcinoma cells. The suppression of A2780 and Caov-3 cell proliferation was maximum at $48 \mathrm{~h}$ of pleuromutilin treatment in the range of $20-200 \mu \mathrm{M}$. At $200 \mu \mathrm{M}$ of pleuromutilin, 


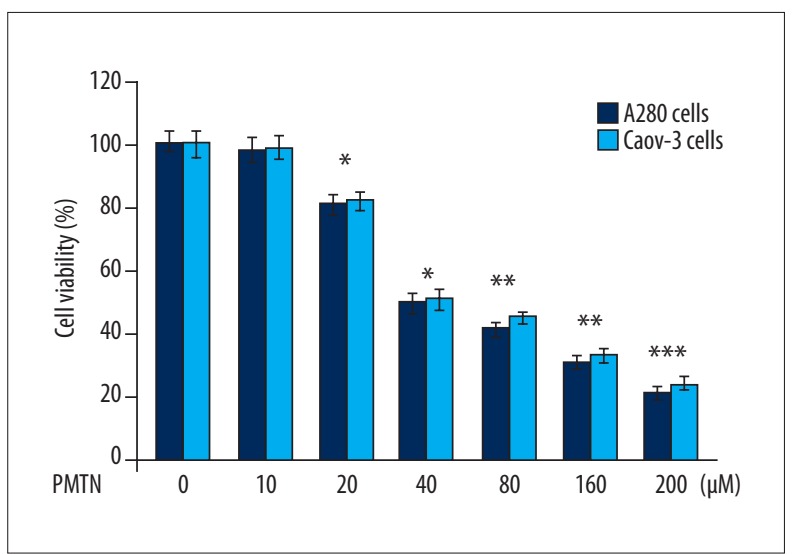

Figure 2. A2780 and Caov-3 human ovarian carcinoma cell proliferation were reduced by pleuromutilin. The cells were treated with 0-200 $\mu \mathrm{M}$ concentrations of pleuromutilin. The Edu proliferation assay was used to evaluate cell proliferation and cell cytotoxicity. ${ }^{*} \mathrm{P}<0.05,{ }^{* *} \mathrm{P}<0.02$ and ${ }^{* * *} \mathrm{P}<0.01$ vs. the untreated cells.

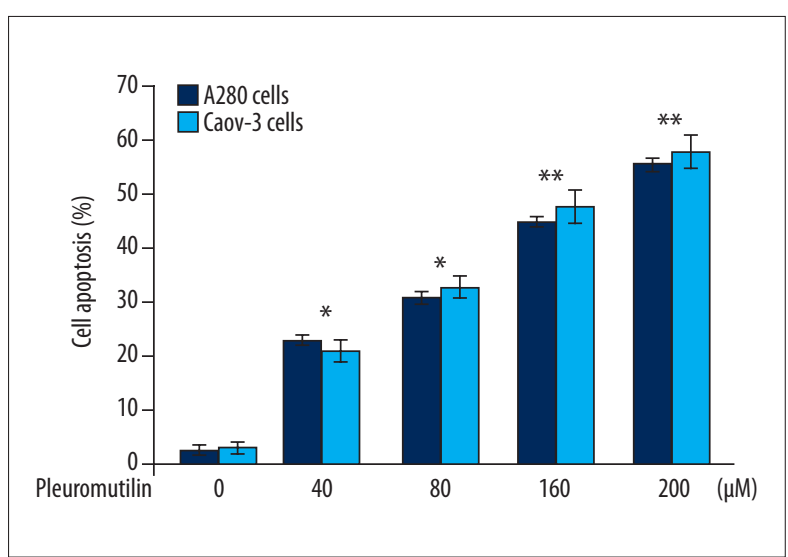

Figure 3. The effect of pleuromutilin on A2780 and Caov-3 human ovarian carcinoma cell apoptosis. The cells after treatment with pleuromutilin at different concentrations were analyzed by flow cytometry. Cell apoptosis was quantified. ${ }^{*} \mathrm{P}<0.05$ and ${ }^{* *} \mathrm{P}<0.01$ vs. untreated cells.

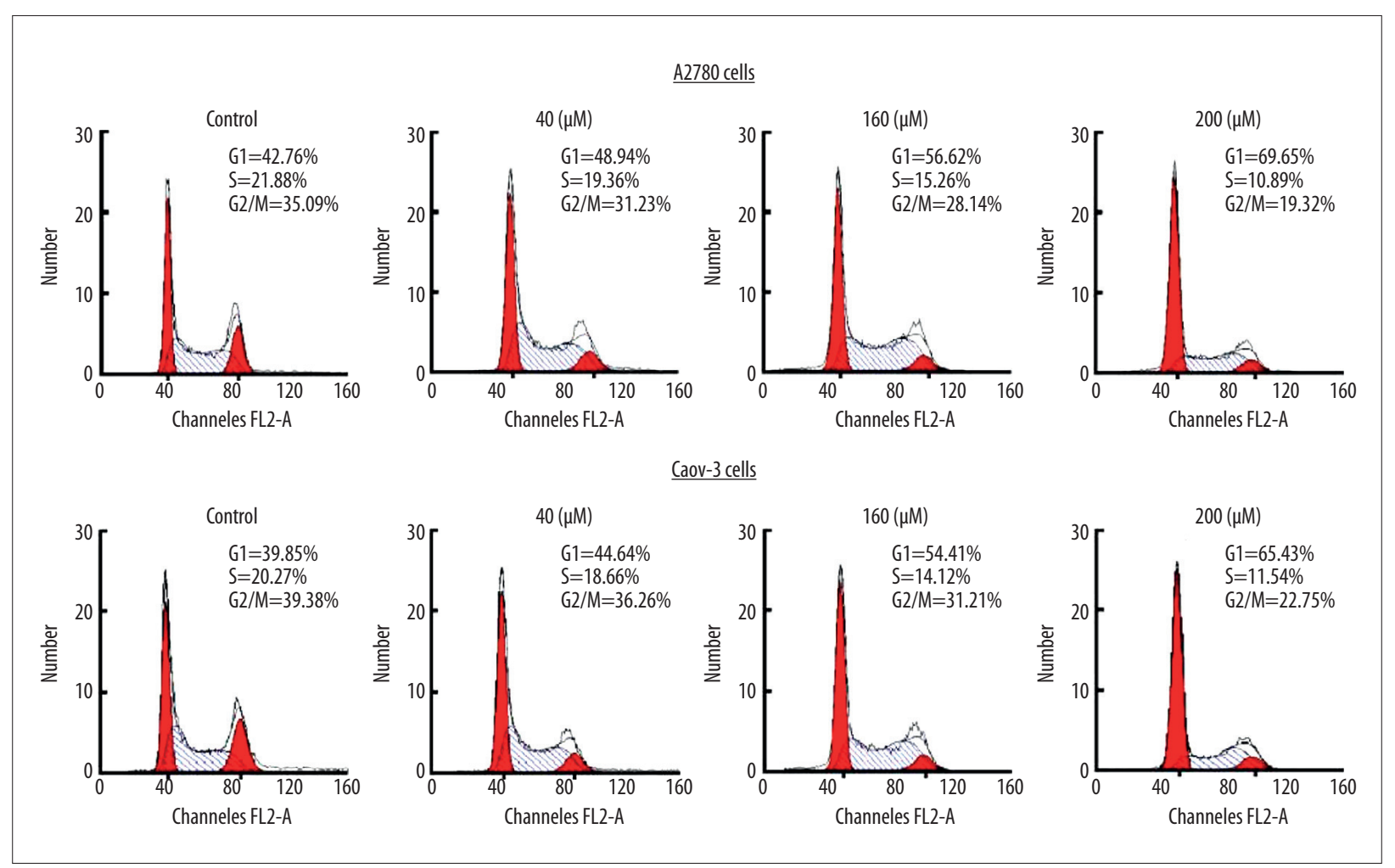

Figure 4. The effect of pleuromutilin on A2780 and Caov-3 human ovarian carcinoma cell cycle progression. The cells were treated with increasing concentrations of pleuromutilin and then analyzed by flow cytometry. 


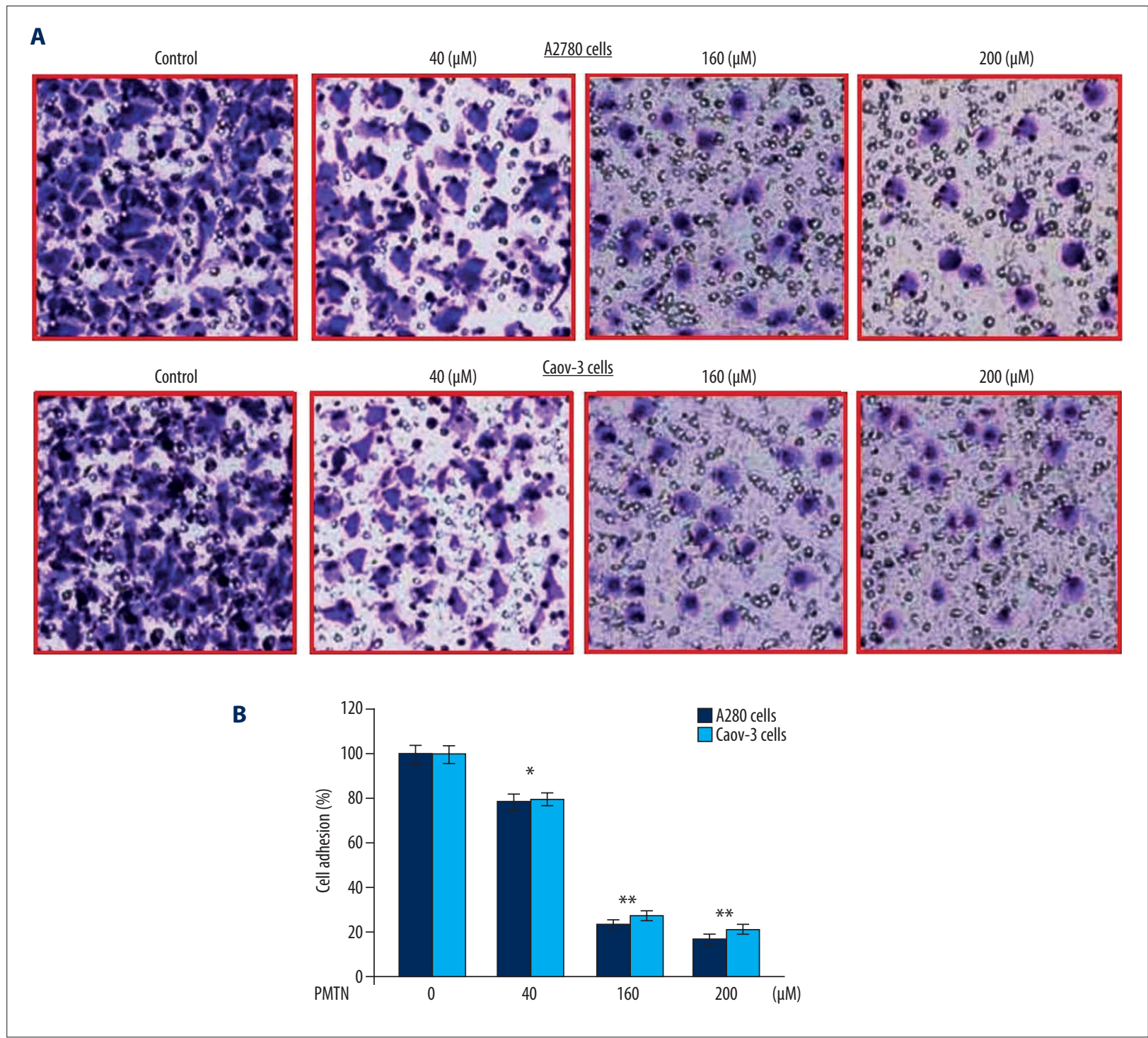

Figure 5. The effect of pleuromutilin on A2780 and Caov-3 human ovarian carcinoma cell adhesion. (A) The cells after treatment with pleuromutilin at increasing concentrations were analyzed for adhesion. Magnification $\times 200$. (B) The population of cell adhesion was quantified. ${ }^{*} \mathrm{P}<0.05,{ }^{* *} \mathrm{P}<0.02$ and ${ }^{* * *} \mathrm{P}<0.01$ vs. the untreated cells.

the proliferation of A2780 and Caov-3 cells was reduced to 21.43 and $23.65 \%$, respectively.

\section{Pleuromutilin induced apoptosis in A2780 and Caov-3 cells}

Exposure of A2780 and Caov- 3 cells to pleuromutilin for $48 \mathrm{~h}$ significantly increased apoptosis (Figure 3). The apoptosis induction in A2780 and Caov-3 cells was significant from $40 \mu \mathrm{M}$ pleuromutilin.

\section{Pleuromutilin resulted in A2780 and Caov-3 cell cycle arrest}

Pleuromutilin treatment significantly increased the A2780 and Caov-3 cell populations in the $\mathrm{G} 1$ phase of the cell cycle when compared with the control (Figure 4). However, the percentage of A2780 and Caov- 3 cells in the S-phase and G2/M phase was significantly reduced following treatment with pleuromutilin for $48 \mathrm{~h}$.

\section{Pleuromutilin reduced $\mathrm{A} 2780$ and Caov-3 cell adhesion}

Treatment of A2780 and Caov-3 cells with pleuromutilin for $48 \mathrm{~h}$ caused a significant reduction in cell adhesion in a dosedependent manner (Figure 5). A2780 and Caov-3 cell adhesion was suppressed by $18 \%$ and $23 \%$, respectively, on treatment with $200 \mu \mathrm{M}$ of pleuromutilin when compared with the untreated cells. 

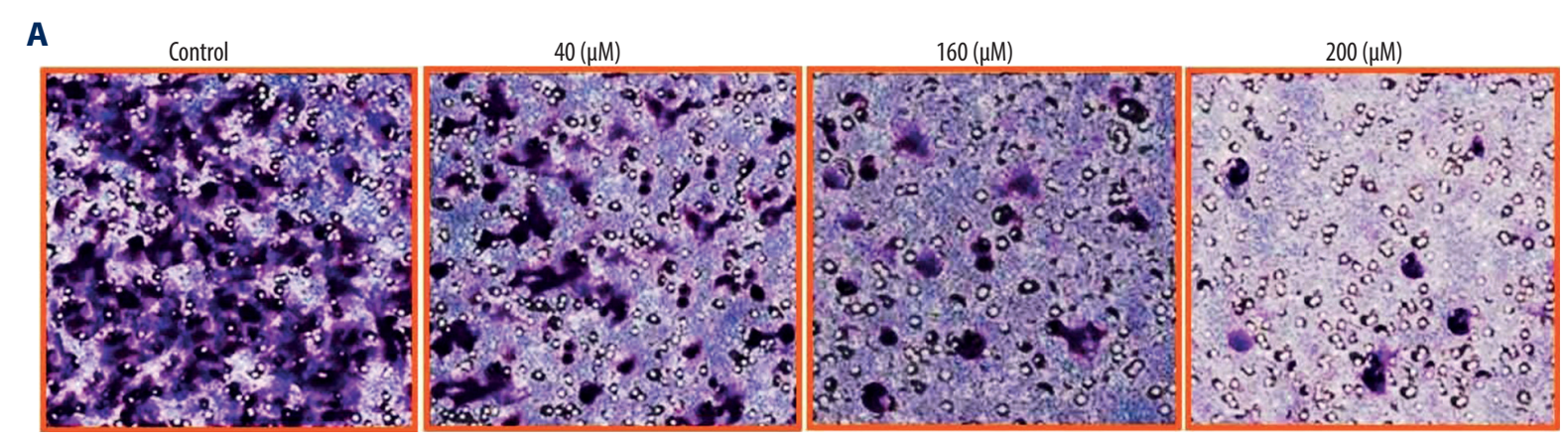

B



Figure 6. The effect of pleuromutilin on A2780 human ovarian carcinoma cell invasion. (A) The cells treated with pleuromutilin for $48 \mathrm{~h}$ were examined for invasion by the transwell assay. Magnification $\times 200$. (B) The results were quantified. ${ }^{*} \mathrm{P}<0.05$ and ${ }^{* *} \mathrm{P}<0.02$ vs. the untreated cells.

\section{Pleuromutilin reduced A2780 cell invasion}

The cell invasion assay showed a significant $(P<0.02)$ reduction in A2780 cell invasion by treatment with pleuromutilin (Figure 6). An ncrease in pleuromutilin concentration from 40 to $200 \mu \mathrm{M}$ resulted in reduced $\mathrm{A} 2780$ cell invasion compared with the untreated cells.

\section{Pleuromutilin reduced $\mathbf{A 2 7 8 0}$ cell migration}

The migration of A2780 cells was suppressed significantly on treatment with pleuromutilin (Figure 7). The wound-healing assay showed that pleuromutilin treatment at $200 \mu \mathrm{M}$ almost completely inhibited A2780 cell migration compared with the untreated cells.

\section{Regulation of p-FAK, MMP-2 and MMP-9 expression by pleuromutilin}

In A2780 cells, pleuromutilin treatment decreased the expression of $\mathrm{p}$-FAK in a dose-dependent manner (Figure 8). The reduction of p-FAK expression was significant from $20 \mu \mathrm{M}$ of pleuromutilin at $48 \mathrm{~h}$. The expression of MMP-2 and MMP-9 were also suppressed in pleuromutilin treated A2780 cells compared with the control. Although the reduction of MMP-2 and MMP-9 expression by pleuromutilin was significant from $20 \mu \mathrm{M}$, but the effect was maximum at $200 \mu \mathrm{M}$.

\section{Inhibition of in vivo tumor metastasis by pleuromutilin}

In the mouse ovarian tumor xenograft model treated with pleuromutilin, the tumor size was significantly less when compared with the untreated group (Figure 9). The tumor size in untreated, $50 \mathrm{mg} / \mathrm{kg}, 100 \mathrm{mg} / \mathrm{kg}, 150 \mathrm{mg} / \mathrm{kg}$ and $100 \mathrm{mg} / \mathrm{kg}$ pleuromutilin treatment groups was $1621.5 \pm 400.6,1543.8 \pm 360.3$, $1476.2 \pm 305.7,1225.6 \pm 280.5$ and $814.9 \pm 190.6 \mathrm{~mm}^{3}$, respectively. In the untreated mouse group, ovarian xenograft tumor metastasis occurred to the intestine, spleen, and peritoneal cavity. Treatment of the mouse xenograft tumor model with pleuromutilin inhibited metastases to the intestine, spleen, and peritoneal cavity. All the mice in the $200 \mathrm{mg} / \mathrm{kg}$ pleuromutilin treatment group survived during the study. however, the survival rate of mice in the treated, $50 \mathrm{mg} / \mathrm{kg}, 100 \mathrm{mg} / \mathrm{kg}$ and $150 \mathrm{mg} / \mathrm{kg}$ treatment groups were $60 \%, 80 \%, 90 \%$, and $100 \%$, respectively.

\section{Discussion}

The aim of this study was to investigate the effects of pleuromutilin on migration and proliferation of A2780 and Caov-3 


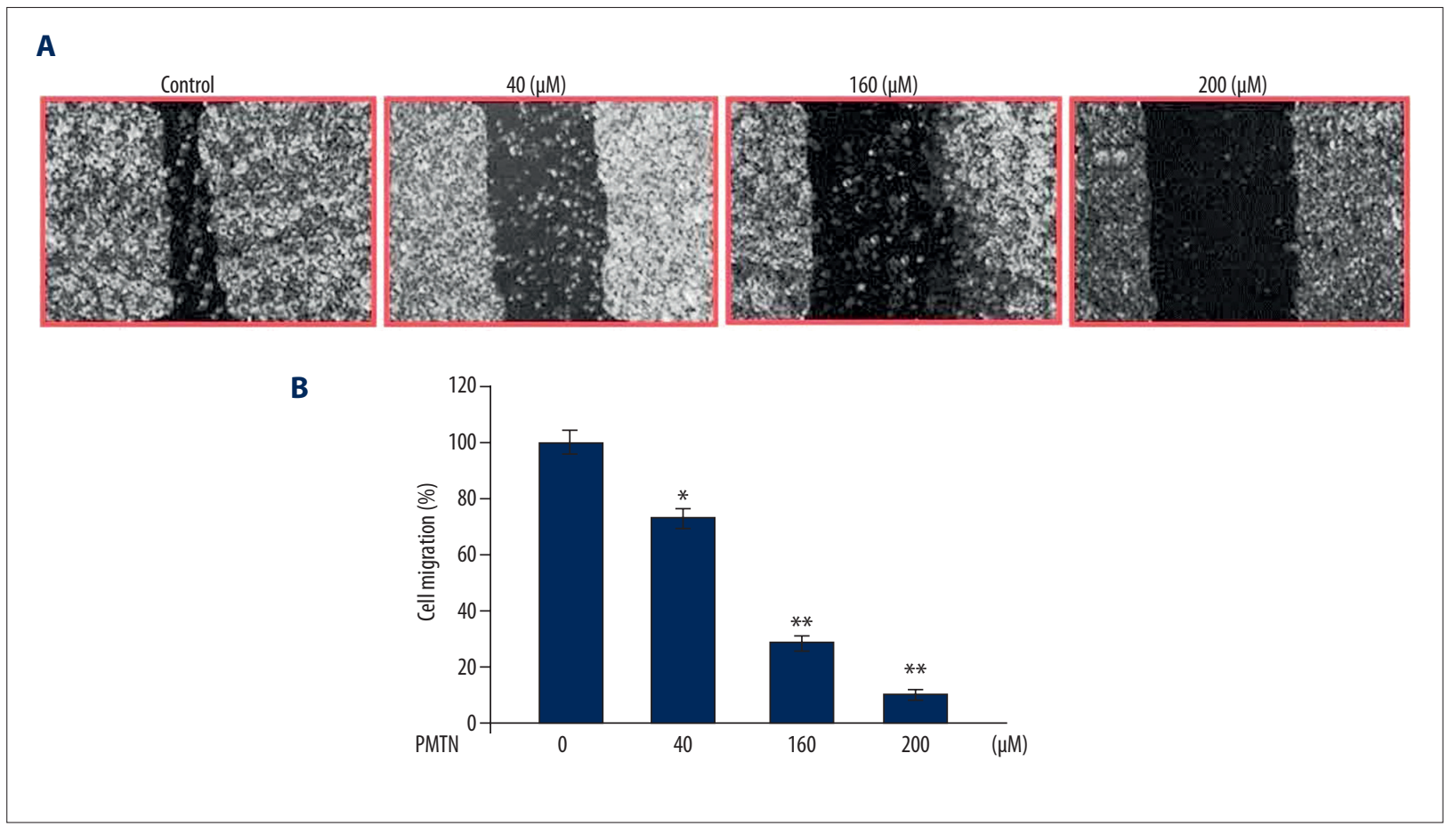

Figure 7. The effect of pleuromutilin on A2780 human ovarian carcinoma cell migration. (A) The cells were treated with increasing concentrations of pleuromutilin and examined by phase-contrast microscopy. Images were taken at a magnification of $\times 200$. (B) Cell migration was quantified. ${ }^{*} \mathrm{P}<0.05$ and ${ }^{* *} \mathrm{P}<0.02$ vs. the untreated cells.

A

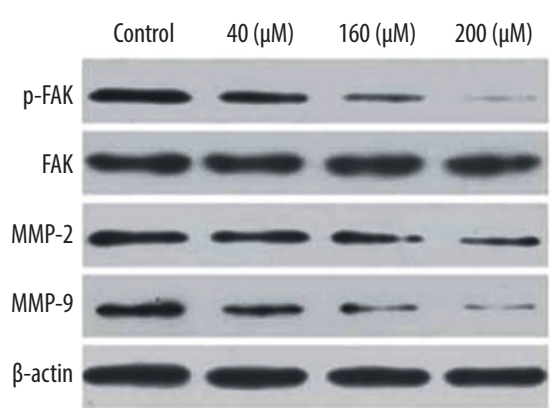

\section{B}

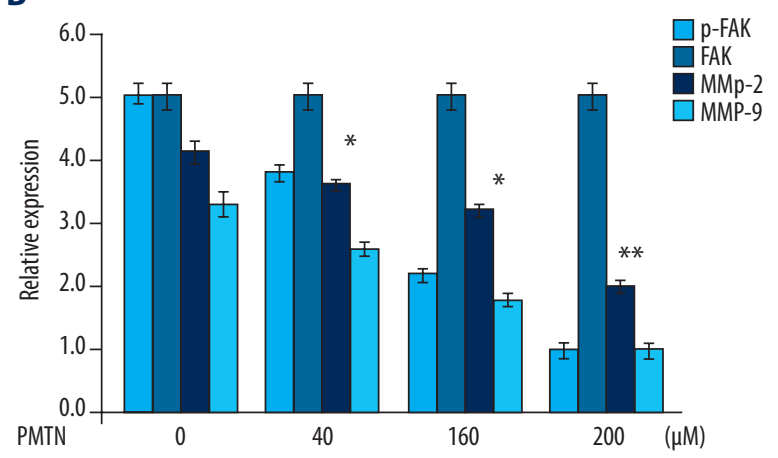

Figure 8. The effect of pleuromutilin on p-FAK and matrix metalloproteinases in A2780 human ovarian carcinoma cells.

(A) The expression of p-FAK, MMP-2, and MMP-9 in A2780 cells at $48 \mathrm{~h}$ of pleuromutilin treatment assessed by Western blot. (B) Relative expression of p-FAK, MMP-2, and MMP-9 in A2780 cells. The level of $\beta$-actin used as control.

${ }^{*} \mathrm{P}<0.05$ and ${ }^{* *} \mathrm{P}<0.01$ vs. the untreated cells.

human ovarian carcinoma cells and the growth of A2780 tumor xenografts in mice. The findings showed that pleuromutilin significantly reduced ovarian cancer cell proliferation in vitro by decreasing the proliferation, metastasis, and adhesion through the down-regulation of FAK activation and MMP-2/9 expression. A2780 tumor xenograft growth and metastasis were also inhibited by pleuromutilin treatment in mice.
Tumor cell adhesion, metastasis, and angiogenesis play a vital role in the development and progression of malignancy $[19,20]$. Detached tumor cells adhere to sites of metastasis by receptors present in the cell membranes $[19,20]$. The high rate of proliferation of these cells is the basis of the pathogenesis of cancer [19]. In the present study, pleuromutilin treatment significantly reduced $\mathrm{A} 2780$ and Caov-3 cell proliferation in a dosedependent manner. Treatment with pleuromutilin significantly 


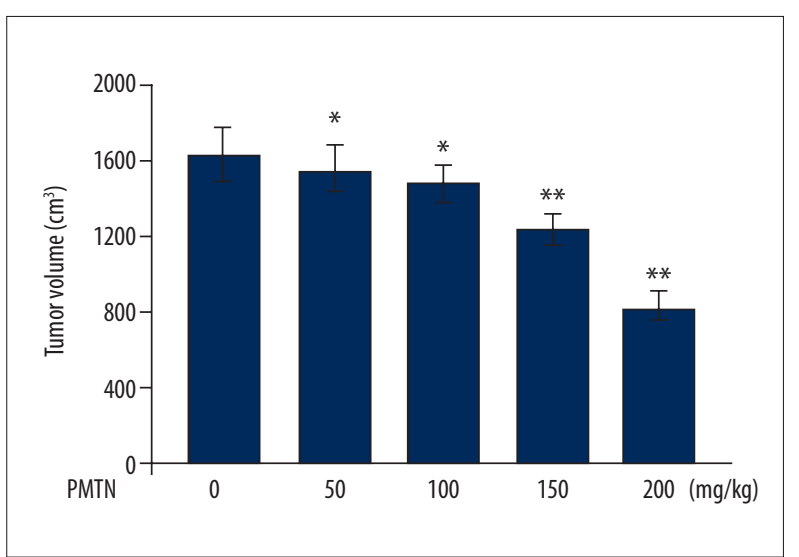

Figure 9. The effect of pleuromutilin on A2780 mouse xenograft tumor growth and metastasis. The mice were inoculated with A2780 cells and treated with increasing doses of pleuromutilin. The volume of tumors excised from the mice was measured on day 31 after $\mathrm{A} 2780$ cell inoculation. ${ }^{*} \mathrm{P}<0.05$ and ${ }^{* *} \mathrm{P}<0.02$ vs. the untreated mice.

suppressed A2780 and Caov-3 cell adhesion when compared with the untreated cells. The migration and invasion of A2780 cells treated with pleuromutilin were significantly reduced compared with the control cells. These findings suggested that pleuromutilin had tumor suppressor effects in A2780 human ovarian carcinoma cells in vitro.

Carcinoma cell metastasis and adhesion is regulated by several pathways through the expression of molecules that in clude MMPs, TIMPs, and FAK $[21,22]$. FAK has a vital role in the regulation of interactions between cells and the extracellular matrix (ECM) $[20,21]$. Phosphorylation of FAK promotes the migration of malignant cells, increases cell proliferation, and cell survival $[22,23]$. The results from the present study showed that pleuromutilin had an inhibitory effect on the phosphorylation of FAK in A2780 cells. These findings suggest that

\section{References:}

1. Siegel RL, Miller KD, Jemal A: Cancer statistics, 2016. Cancer J Clin, 2016; 66: 7-30

2. Bertone-Johnson ER: Epidemiology of ovarian cancer: A status report. Lancet, 2005; 365: 101-2

3. Kurman RJ, Shih leM: The origin and pathogenesis of epithelial ovarian cancer: A proposed unifying theory. Am J Surg Pathol, 2010; 34: 433-43

4. Jemal A, Siegel R, Ward E et al: Cancer statistics, 2009. Cancer J Clin, 2009; 59: $225-49$

5. Kroeger PT Jr., Drapkin R: Pathogenesis and heterogeneity of ovarian cancer. Curr Opin Obstet Gynecol, 2017; 29: 26-34

6. Saijo N: Present status and problems on molecular targeted therapy of cancer. Cancer Res Treat, 2012; 44: 1-10

7. Ye W, Zhiling Z: Oridonin inhibits metastasis of human ovarian cancer cells by suppressing the mTOR pathway. Arch Med Sci, 2019; 15(4): 1017-27 pleuromutilin suppressed the metastatic potential of A2780 cells by targeting FAK activation.

Carcinoma cell metastasis through the basement membrane and extracellular matrix (ECM) is increased by the overexpression of matrix metalloproteinases (MMPs) [19,21,24]. Previous studies have shown a direct relationship between the expression of MMPs and carcinoma cell metastasis $[19,25,26]$. In the present study, pleuromutilin treatment significantly suppressed the expression of MMP-2/9 in A2780 cells at $48 \mathrm{~h}$. Therefore, pleuromutilin treatment inhibited the proliferation and metastasis of A2780 cells by targeting MMP overexpression. Ovarian carcinoma metastasizes to distant organs that include the lungs [27], pancreas [28], peritoneum [29] and hepatic tissues [30]. The present study investigated the effect of pleuromutilin on the development of mouse A2780 tumor xenografts and metastasis in vivo. The results showed that pleuromutilin treatment of mouse tumor xenografts inhibited tumor growth and metastasis in a dose-dependent manner. The in vivo effects of pleuromutilin in this study were not associated with toxicity.

\section{Conclusions}

This study aimed to investigate the effects of pleuromutilin on migration and proliferation of A2780 and Caov-3 human ovarian carcinoma cells and the growth of A2780 tumor xenografts in mice and the molecular mechanisms involved. In A2780 and Caov-3 human ovarian carcinoma cells, pleuromutilin inhibited cell proliferation, migration, invasion, and adhesion in a dose-dependent manner, and reduced tumor growth and metastases in a mouse A2780 cell tumor xenograft model.

\section{Conflict of interest}

None.

8. Thompson N, Adams DJ, Ranzani M: Synthetic lethality: Emerging targets and opportunities in melanoma. Pigment Cell Melanoma Res, 2017; 30 : 183-93

9. Duan P, Fan L, Gao $Q$ et al: Targeted therapy of ovarian cancer with angiogenesis inhibitors. Curr Drug Targets, 2017; 18: 1171-78

10. Hamanishi J, Mandai M, Ikeda T et al: Safety and antitumor activity of anti-PD-1 antibody, nivolumab, in patients with platinum-resistant ovarian cancer. J Clin Oncol, 2015; 33: 4015-22

11. Kavanagh F, Hervey A, Robbins WJ: Antibiotic substances from basidiomycetes: VIII. Pleurotus multilus (Fr.) sacc. and Pleurotus passeckerianus pilat. Proc Natl Acad Sci USA, 1951; 37(9): 570-74.

12. Drews J, Georgopoulos A, Laber $G$ et al: Antimicrobial activities of 81.723 hfu, a new pleuromutilin derivative. Antimicrob Agents Ch, 1975; 7: 507-16

13. Hogenauer G: Mode of action of pleuromutilin derivatives - location and properties of pleuromutilin binding-site on Escherichia-coli ribosomes. Eur J Biochem, 1975; 52: 93-98 
14. Llabani E, Hicklin RW, Lee HY et al: Diverse compounds from pleuromutilin lead to a thioredoxin inhibitor and inducer of ferroptosis. Nat Chem, 2019; 11(6): 521-32

15. Egger $\mathrm{H}$, Reinshagen $\mathrm{H}$ : New pleuromutilin derivatives with enhanced antimicrobial activity. I. Synthesis. J Antibiot, 1976; 29: 915-22

16. Egger $\mathrm{H}$, Reinshagen $\mathrm{H}$ : New pleuromutilin derivatives with enhanced antimicrobial activity. 2. Structure-activity correlations. J Antibiot, 1976; 29: 923-27

17. Hunt E: Pleuromutilin antibiotics. Drugs Future, 2000; 25: 1163-68

18. Schluenzen F, Pyetan E, Fucini $P$ et al: Inhibition of peptide bond formation by pleuromutilins: The structure of the $50 \mathrm{~S}$ ribosomal subunit from Deinococcusradiodurans in complex with tiamulin. Mol Microbiol, 2004; 54: 1287-94

19. Zhang J, Wang B: Arsenic trioxide $(\mathrm{As}(2) \mathrm{O}(3))$ inhibits peritoneal invasion of ovarian carcinoma cells in vitro and in vivo. Gynecol Oncol, 2006; 103: 199-206

20. Cattaruzza S, Perris R: Proteoglycan control of cell movement during wound healing and cancer spreading. Matrix Biol, 2005; 24: 400-17

21. Hoekstra R, Eskens FA, Verweij J: Matrix metalloproteinase inhibitors: Current developments and future perspectives. Oncologist, 2001; 6: 415-27

22. Parsons JT, Martin KH, Slack JK et al: Focal adhesion kinase: A regulator of focal adhesion dynamics and cell movement. Oncogene, 2000; 19: 5606-13
23. Provenzano PP, Keely PJ: The role of focal adhesion kinase in tumor initiation and progression. Cell Adh Migr, 2009; 3: 347-50

24. Anfosso L, Efferth T, Albini A, Pfeffer U: Microarray expression profiles of angiogenesis-related genes predict tumor cell response to artemisinins. Pharmacogenomics J, 2006; 6: 269-78

25. Fatima ASA: The role of matrix metalloproteinases in cancer progression, in particular metastasis. Arch Med Sci Civil Dis, 2018; 3: e124-46

26. Weronika LG, Artur W-R, Janusz $S$ et al: Matrix metalloproteinases, inhibitor of metalloproteinases mRNA and protein expression in laryngeal squamous cell carcinoma. Arch Med Sci, 2019; 15(3): 784-91

27. Zhou HJ, Zhang JL, Li A et al: Dihydro-artemisinin improves the efficiency of chemotherapeutics in lung carcinomas in vivo and inhibits murine Lewis lung carcinoma cell line growth in vitro. Cancer Chemother Pharmacol, 2010; 66: $21-29$

28. Wang SJ, Gao Y, Chen $\mathrm{H}$ et al: Dihydroartemisinin inactivates NF-kappaB and potentiates the anti-tumor effect of gemcitabine on pancreatic cancer both in vitro and in vivo. Cancer Lett, 2010; 293: 99-108

29. Disbrow GL, Baege AC, Kierpiec KA et al: Dihydroartemisinin is cytotoxic to papillomavirus-expressing epithelial cells in vitro and in vivo. Cancer Res, 2005; 65: 10854-61

30. Hou J, Wang D, Zhang R, Wang H: Experimental therapy of hepatoma with artemisinin and its derivatives: In vitro and in vivo activity, chemosensitization, and mechanisms of action. Clin Cancer Res, 2008; 14: 5519-30 